\title{
ABBV-744 and Onalespid as Potential Inhibitors of SARS-CoV-2 main Protease Enzyme: A Promising Therapeutics in COVID-19?
}

\section{Zeynab Fakhar}

University of the Witwatersrand

\section{Shama Khan}

University of the Witwatersrand

Aijaz Ahmad ( $\square$ aijaz.ahmad@wits.ac.za)

University of the Witwatersrand

\section{Research Article}

Keywords: Main protease inhibitor; SARS-CoV-2; COVID-19; 3D-Pharmacophore based structure modeling; Docking based virtual screening workflow; ADMET; Molecular dynamics simulations

Posted Date: June 25th, 2020

DOI: https://doi.org/10.21203/rs.3.rs-37619/v1

License: (9) This work is licensed under a Creative Commons Attribution 4.0 International License. Read Full License 


\section{Abstract}

A new pathogen severe acute respiratory syndrome coronavirus 2 (SARS-CoV-2) has spread worldwide and become pandemic with thousands new deaths and infected cases globally. To address coronavirus disease (COVID-19), currently no effective drug or vaccine is available. This necessity motivated us to explore potential lead compounds by considering drug repurposing approach targeting main protease $\left(\mathrm{M}^{\mathrm{pro}}\right)$ enzyme of SARS-CoV-2. This enzyme considered to be an attractive drug target as it contributes significantly in mediating viral replication and transcription. Herein, comprehensive computational investigations were performed to identify potential inhibitors of SARS-CoV-2 $\mathrm{M}^{\mathrm{pro}}$ enzyme. The structurebased pharmacophore modeling was developed based on the co-crystallized structure of the enzyme with its biological active inhibitor. The generated hypotheses were applied for virtual screening based PhaseScore. Docking based virtual screening work-flow was used to generate hit compounds using HTVS, SP and XP based Glide GScore. The pharmacological and physicochemical properties of the best hit compounds were characterized using ADMET. Molecular dynamics simulations were performed to explore the binding affinities of the considered compounds. Binding studies revealed that compound ABBV-744 binds to the $M^{\text {pro }}$ with strong affinity $\left(\square G_{\text {bind }}-45.43 \mathrm{kcal} / \mathrm{mol}\right)$, and the complex is more stable in comparison with other protein-ligand complexes. Our study classified three best compounds which could be considered as promising inhibitors against main protease SARS-CoV-2 virus.

\section{Highlights}

- SARS-CoV-2 disclosed a close genetic resemblance to the severe acute respiratory syndrome coronavirus (SARS-CoV) that already triggered an epidemic in 2003.

- The main protease $\left(\mathrm{M}^{\mathrm{pro}}\right)$ is a quintessential enzyme which contributes significantly in the life cycle of SARS-CoV-2 and inhibition of $\mathrm{M}^{\text {pro }}$ enzyme activity would block viral replication.

- Herein, comprehensive computational investigations were performed to identify potential inhibitors of SARS-CoV-2 $\mathrm{M}^{\text {pro }}$

\section{Introduction}

Coronavirus disease 2019 (COVID-2019) outbreak is a global pandemic caused by severe acute respiratory syndrome coronavirus 2 (SARS-CoV-2) which initially diagnosed in Chinese patients of Hubei's Wuhan city in early December 2019 [1]. SARS-CoV-2 disclosed a close genetic resemblance to the severe acute respiratory syndrome coronavirus (SARS-CoV) that already triggered an epidemic in 2003 [2]. COVID-19 has been declared a global health disaster by World Health Organization (WHO) on 30 January 2020 as the disease hastily transmitted human-to-human and affected more than 170 countries across the world [3]. The existing condition is extremely increasing; therefore, the overall asperity of this disease persist to be serious. The infection rate of SARS-CoV-2 is higher (10-12\%) in comparison with its mortality 
rate (5.4\%) [4]. The most distinctive indications of COVID-19 patients are high fever, cough and excessive respiratory sickness that required urgent intensive care facility. Currently, there is no applicable and precise medication for the treatment of COVID-19, however, many drugs and vaccines are under clinical trials. The only practical approach available is the repurposing of existing antiviral drugs as these drugs have already been tested for their toxicity [5]. Still there is a prompt requirement to make substantial efforts to advance therapeutic interventions against CoV infections.

CoVs are single-stranded positive-sense RNA viruses belongs to the family of Coronaviridae. These viruses can be categorized into four species: alpha, beta, gamma and delta. The recent SARS-CoV-2 is from beta genus and is usually identified to affect commonly humans [6]. The RNA genome length of this virus is about $27-32 \mathrm{~Kb}$ encoding both structural and non-structural proteins. The structural proteins are membrane (M), envelope (E), nucleocapsid $(N)$, hemagglutinin-esterase $(H E)$ and spike (S) proteins contribute notably in viral transmission and replication in the host cells [7]. Among which $3 \mathrm{C}$-like protease (3CLpro) protease plays critical role in virus replication and transcription, thus studied as potential drug targets. Providing informative knowledge regarding the enzyme inhibition will be valuable in tailoring effective and selective inhibitors of 3CLpro that will ultimately be associated to report COVID-19 as this protease is imperative for virus assembly and reproduction [8].

The main protease $\left(\mathrm{M}^{\text {pro }}\right)$ is a quintessential enzyme which contributes significantly in the life cycle of SARS-CoV-2 and inhibition of $M^{\text {pro }}$ enzyme activity would block viral replication. Since no human proteases with a similar specified cleavage are characterized, thus the potential inhibitors are unlikely to be toxic. The $\mathrm{M}^{\mathrm{pro}}$ enzyme consists of an asymmetric unit including 306 amino acid residues with CYS145 and HIS41 catalytic dyad in the active site [9][10] (Figure 1).

Experimental observations by Zhang et al. [10] demonstrated the half maximal inhibitory concentration (IC50) value $0.67 \mathrm{mM}$ for an a-ketoamide inhibitor (ak-13b) as a potent antiviral inhibitor against $\mathrm{M}^{\text {pro }}$ enzyme. Zhang et al. proposed the inhibition mechanism through the nucleophilic attack of the catalytic CYS145 to the a-keto group of the inhibitor forming thiohemiketal. This thiohemiketal group is stabilized by formation of several hydrogen bonds with the active residues of HIS41, GLY143, SER144 and CYS145, Figure 1. This experimental observation proposed that a-ketoamide inhibitors interacted with the catalytic center of proteases through two hydrogen bond interactions whereas aldehydes and Michael acceptors only formed one hydrogen bond into the catalytic center of the target proteases [11][12].

According to the aforementioned experimental structural information, we have chosen SARS-CoV-2 $\mathrm{M}^{\text {pro }}$ as a target enzyme to accelerate the prompt hunt of antiviral drug repurposing with clinical potential to gain a short-term and specific solution to treat COVID-19 patients.

To address this challenge, an specific library of anti and pro-viral agents including FDA approved drugs, compounds in clinical trials and preclinical compounds having inhibitory activity between 10-100 nM range against SARS-CoV-2 was considered for drug repurposing to attain immediate and precise results [13]. In this study, we developed an integrated approach of drug discovery integrating 3D structure-based 
pharmacophore modelling, virtual screening of the considered library, molecular docking workflow, ADMET pharmacological analysis and molecular dynamics (MD) simulations to find potential antiviralagents for the therapeutic management of COVID-19. This scheme will provide an informative insight into the exploration of potent antiviral drugs which, could help in progressive attempts in the therapeutics of COVID-19.

\section{Methodology}

\subsection{System preparation}

The $1.95 \AA$ crystal structure of SARS-CoV-2 main protease $\left(M^{\text {pro }}\right)$ in complex with inhibitor was extracted from the Protein Data Bank (PDB ID: 6Y2F) [10]. The structure of the enzyme was pre-processed, minimized and refined using the Protein Preparation Wizard [14] implemented in Schrödinger suite. This involved eliminating crystallographic waters, missing hydrogens/side chain atoms were added, and the appropriate charge and protonation state was assigned to the receptor structure corresponding to $\mathrm{pH} 7.0$ considering the appropriate ionization states for the acidic as well as basic amino acid residues.

Structure was subjected to energy minimization using the OPLS-2005 force-field [15][16] with a root mean square deviation (RMSD) cut-off value of $0.30 \AA$ to relieve the steric clashes among the residues due to the addition of hydrogen atoms.

The preparation of the crystalized inhibitor, ak-13b and the candidate compounds were achieved using LigPrep module of Schrodinger Suite which undertakes hydrogens atom addition, amending realistic bond lengths and angles, accurate chiralities, ionization states, tautomers, stereo chemistries, and ring conformations. Partial charges were assigned to the structures using the OPLS-2005 force-field [15] and the subsequent structures were imperiled to energy minimization until their average RMSD reached 0.001 $\AA$. The ionization state was set at the neutral pH using Epik ionization tool [17].

\subsection{Preparation of Inhibitor-like Ligand Library}

We have retrieved the 75 candidate compounds by Gordon et al. [13] as these compounds were selected based on their experimental anti-viral activity against SARS-CoV-2 of coronavirus. The total 75 compounds were considered for further virtual screening analysis. The list of the compounds has been displayed in Table S1.

\subsection{Identification of 3D-Pharmacophore Hypotheses}

For the structure-based pharmacophore modeling Schrodinger PHASE module [18] was used with the default set of seven chemical features- hydrogen bond acceptor (A), hydrogen bond donor (D), hydrophobic contacts $(H)$, negative ionizable $(N)$, positive ionizable $(P)$, and aromatic ring $(R)$ to create the utmost illustrative features of the $\mathrm{M}^{\text {pro }}$ active sites. The seven 3D-features were generated using Hypothesis Generation for Energy-Optimized Structure Based Pharmacophores considering the omitted volumes within $5 \AA$ of the refined ligand for the enzyme [19]. Pharmacophore features were chosen based 
on the crucial interactions with the key residues of the enzyme accommodated the inhibitor. The resulted pharmacophore features comprise the functional groups included in their bioactivity of targeted enzyme. The omitted volumes contain all atoms within $5 \AA$ of the refined ligand for the target.

\subsection{Screening of $M^{\text {pro }}$ Inhibitors}

All acquired seven 3D-pharmacophore features were exported and used for PHASE-based virtual screening to screen the candidate library of 75 compounds which were retrieved from Gordon et al[13] recent experimental work. Out of 75 compounds, 43 were generated based on the highest PHASE screen score and matched ligand sites (Table S2). Both the quantity and quality of feature matching is taken into account in the Phase-Screen-Score factor.

\subsection{Docking-based virtual screening}

Molecular-docking-based virtual screening was performed using Glide-Based virtual screening workflow of Maestro 11.6 to prioritize the hit candidate compounds that strongly bind to $\mathrm{M}^{\text {pro }}$ enzyme [20]. Receptor grid was created as center coordinates $(X=9.81 Y=-1.47 Z=20.51)$ using two cubic boxes having a mutual centroid to systematize the calculations: a larger enclosing and a smaller binding box with dimensions of $24 \times 24 \times 24 \AA$ and $18 \times 18 \times 18 \AA$, correspondingly. The grid box was centered on the centroid of the ligands in the complex, which was adequately large enough to search a superior region of the enzyme structure. All the chosen ligands were docked by using a three docking protocols of Glide [20] which starts with "High throughput Virtual Screening" (HTVS) followed by "Standard Precision" (SP) and then by "Extra-Precision" mode (XP). Finally, 43 input compounds were assessed using Docking-Based Virtual Screening and filtered to final three optimized lead compounds based on XP-GScores.

\subsection{ADMET Properties Assessment}

Schrodinger QikProp 5.6 module was used to calculate absorption, distribution, metabolism, excretion and toxicity (ADMET) properties of the considered compounds to produce the ADMET associated descriptors. This protocol predicts noteworthy physicochemical and pharmacokinetic-based descriptors of the compounds based on Lipinski's rule of five [21][22]. ADMET properties of the top three compounds and one control inhibitor were considered and studied using QikProp 5.6 module and the best three compounds were chosen for final molecular dynamics (MD) simulations.

\subsection{Simulations}

MD simulation considered to be the most essential approach in understanding the fundamental structure and function of biological macromolecules. This method helps in finding the underlying dynamics and how it is connected to enzyme's biomolecular function [23][24][25]. AMBER 18 [26] simulation package was used to execute $200 \mathrm{~ns}$ MD simulations on all the prepared complexes using (Graphics Processing Unit) GPU accelerated version of Partial Mesh Ewald Molecular Dynamics (PMEMD) simulations [27]. The 
ff99SB [28] and the general AMBER force fields (GAFF) [29][30] were employed to parametrize the enzyme and the considered ligands using LEaP implemented in Amber 18.

The ANTECHAMBER module was used to assign atomic partial charges for the ligands employed in General Amber Force-Field (GAFF). The system was solvated using the TIP3P [31] explicit water in a cubic box with $8 \AA$ box edge. The $\mathrm{Na}^{+}$counter ions were added to randomly to neutralize the complex. The partial Mesh Ewald (PME) [32] method was used to account the long-range electrostatic forces using cutoff of $12 \AA$, and the SHAKE algorithm [33] was used to constrain all the hydrogen atoms bonds.

Energy minimizations were performed in two stages with 2500 steps of steepest decent minimization followed by 2500 of conjugated gradient to remove the bad contacts. The first stage was followed with a harmonic restraint of $500 \mathrm{kcalmol}^{-1} \mathrm{~A}^{-2}$ on the solute molecule whereas, ions and water molecules were relaxed. On the second stage of minimization the restraints were removed and the whole system was relaxed. Each minimized complex was then gradually heated up from $0 \mathrm{~K}$ to $300 \mathrm{~K}$ for $200 \mathrm{ps}$ to keep the solute using a weak harmonic restraint of $10 \mathrm{kcalmol}^{-1} \mathrm{~A}^{-2}$. The $50 \mathrm{ps}$ density equilibration with weak restraints followed by the 500 ps constant pressure equilibration at $300 \mathrm{~K}$ were performed at constant pressure using Berendsen barostat [34]. Ultimately, the production phase of $200 \mathrm{~ns}$ MD simulation was performed on all the complexes at a constant temperature of $300 \mathrm{~K}$ and constant pressure at $1 \mathrm{~atm}$.

\subsubsection{Post-dynamic trajectories analyses}

The 200 ns MD trajectories were analyzed to calculate the RMSD of $C^{a}$ atoms, root mean square fluctuation (RMSF) of each residue in the complex, radius of gyration $\left(R_{\mathrm{g}}\right)$, solvent accessible surface area (SASA), intramolecular and intermolecular hydrogen bond interactions using CPPTRAJ module [35] implemented in AMBER 18. Molecular visualizations and plotting were conducted using Maestro 11.6 and OriginPro 2018 software [36].

\subsubsection{Principal component analysis (PCA)}

PCA as an important tool for identifying the conformational changes of proteins was carried to describe the residual motions upon inhibitor binding of biomolecular complex [37]. PCA generates highly correlated and anti-correlated fluctuations derived from MD trajectories by applying dimensional reduction [38][39]. The collective motions were studied using the positional covariance matrix $\mathrm{C}$ constructed based on the atomic coordinates and their corresponding eigenvectors. The eigenvalues and eigenvectors are defined as the extent and the direction of motions, respectively [40][41]. By the following equation, the matric elements of the positional covariance matrix $C$ were determined: (see Equation 1 in the Supplementary Files)

where $\mathrm{q}_{\mathrm{i}}$ and $\mathrm{q}_{\mathrm{j}}$ are the cartesian coordinates for the $\mathrm{i}$, jth of $\mathrm{Ca}$ atom, and $\mathrm{N}$ is the number of $\mathrm{Ca}$ atoms. To remove all translational and rotational movements, the average is calculated after superimposition with a reference structure using a least-square fit procedure to excerpt the important motion from MD trajectories [42][43][44]. To derive the eigenvalues and eigenvectors, the symmetric matrix $C$ is 
transformed into a diagonal matrix $\Lambda$ of eigenvalues by an orthogonal coordinate transformation matrix $\mathrm{T}$ : (see Equation 2 in the Supplementary Files)

in which the eigenvectors correspond to the direction of motions relative to and each eigenvector associate with an eigenvalue that represents the total mean-square fluctuation of the system along the corresponding eigenvector. CPPTRAJ module from the Amber 18 suite was used to perform the PC analysis and the porcupine plot of protein collective motions was created by NMWiz implemented inVMD [45].

\subsubsection{Binding free energy calculations}

The relative binding free energies were computed using Molecular Mechanics/Generalized Born Surface Area (MM/GBSA) binding free energy method [46]. Water molecules and counter ions were stripped using the CPPTRAJ module. The binding free energies ( $D G_{\text {bind }}$ ) were calculated with the MM-GBSA method for each complex as below: (see Equations 3-8 in the Supplementary Files)

The gas phase energy ( $\left.\mathrm{D} E_{\text {gas }}\right)$ is the sum of the internal $\left(E_{\text {int }}\right)$, van der Waals $\left(E_{\mathrm{vdW}}\right)$ and Coulombic $\left(E_{\text {elec }}\right)$ energies, (Eq. 6). The solvation free energy is the pattern of polar $\left(\mathrm{G}_{\mathrm{GB}}\right)$ and nonpolar (Gnonpolar, solvation) contributions (Eq. 7). The polar solvation $\mathrm{G}_{\mathrm{GB}}$ contribution is estimated using the Generalized Born (GB) solvation model with the dielectric constant 1 for solute and 80.0 for the solvent. Conversely, the nonpolar free energy contribution was assessed using Eq. 8, where the surface tension proportionality constant, $\mathrm{g}$, and the free energy of nonpolar solvation of a point solute, b, were set to $0.00542 \mathrm{kcal} \mathrm{mol}^{-1}$ $\AA^{-2}$ and $0 \mathrm{kcal} \mathrm{mol}^{-1}$, respectively [47]. The SASA is calculated by the linear combination of pairwise overlap (LCPO) model [48].

\section{Results And Discussion}

\subsection{Selection of compounds}

An specific drug repurposing library of 75 anti and pro-viral agents including FDA approved drugs, clinical trials compounds and preclinical compounds having inhibitory activity between 10-100 nM range [13] against SARS-CoV-2 was considered as the input database for this in-silico study, Table S1.

\subsection{Structure-based pharmacophore modeling}

The comprehensive and accurate information of ligand interacting features can be obtained from structure-based pharmacophores based on three-dimensional structure of a target protein [49]. The most common descriptors in pharmacophore modeling are $\mathrm{H}$-bond donors, $\mathrm{H}$-bond acceptors, positive and negative ionizable groups, lipophilic regions and aromatic rings. The most effective 3D structure-based epharmacophores were produced using the receptor-ligand pharmacophore generation protocol implemented in PHASE, which was executed for a co-crystal ligand inside the active pocket in order to determine possibly critical amino acids that are involved in ligand binding (Figure 2A). The generated e- 
pharmacophore for the considered enzyme showed seven main 3D-features including, $\mathrm{H}$-bond acceptor, $\mathrm{H}$-bond donor and aromatic rings. In each pharmacophore model, the red arrows represent hydrogen bond acceptor, blue arrow represents hydrogen bond donor and orange spheres represent an aromatic ring in Figure 2B. Numerous excluded volumes were also produced in the models to demonstrate the space balancing. The 3D pharmacophore features and 2D-chemical structure of $3 \mathrm{~b}$ are presented in Figure 2B and $\mathbf{2 C}$ showing seven 3-D pharmacophore features as three donor hydrogen bonds, three acceptor hydrogen bonds and one aromatic ring sphere.

\subsection{Virtual Screening of the candidate compounds}

The obtained structure-based pharmacophore hypotheses of ak-3b-inhibitor in complex with $\mathrm{M}^{\text {pro }}$ were used to screen the 75 candidate anti-viral compounds retrieved from recent experimental work by Gordon et al [13] (Table S1). These compounds were screened based on PHASE screen score, matched ligand sites indices. A total of 43 compounds passed this filter and subsequently 43 compounds were filtered based on the created pharmacophore hypothesis. Molecules which have satisfied all the features of the pharmacophore model were considered as potential hits. The virtual screened compounds are presented in Table S2.

\subsection{Docking-based virtual screening analysis}

A total of 43 screened compounds obtained from virtual screening were considered for docking analysis using Glide module [50] of Schrödinger package. Three step wise filtering protocol were used for docking using HTVS where a total of 23 compounds (Table S3) were obtained followed by Glide SP where a total of 12 hits were generated (Table S4). Finally, the best five compounds were filtered using Glide XP lead optimization docking while only one pose per ligand was retained (Table S5). The Glide GScore and the interacting binding residues of the five lead compounds presented in Table 1. The ak-13b-inhibitor as well as the best optimized lead generated from XP docking were selected to map their potential interactions within the active pocket of SARS-CoV-2 $\mathrm{M}^{\text {pro }}$ enzyme using molecular docking approach. This approach aids in understanding the optimized orientation of a ligand and its target protein by minimizing inclusive energies of the corresponding complexes. The estimated docking binding energy values of all three compounds and ak-13b-inhibitor are shown in Figure $\mathbf{3}$ and $\mathbf{4}$ and Table $\mathbf{1 .}$

As it is shown in Figure 3, ak-13b-inhibitor interacted to HIS164, GLU166, HIS163, PHE140, ASN 142 and CYS154 through six hydrogen bond interactions. HIS41, CYS44 and MET49 formed three $p$ - p stacking interaction with ak-13b-inhibitor.

The Daunorubicin-M ${ }^{\text {pro }}$, Onalespib-M ${ }^{\text {pro }}$ and ABBV-744-M ${ }^{\text {pro }}$ docked complexes presented noteworthy binding affinities with the energy values of $-9.33,-8.21$ and $-7.79 \mathrm{kcal} \mathrm{mol}^{-1}$, respectively (Table 1 ). These three compounds contributed into the binding site interactions through the non-covalent interactions including hydrogen bond interactions, $\mathrm{p}$ - $\mathrm{p}$ interaction and $\mathrm{p}$-Sulphur interactions present in the binding site of $\mathrm{M}^{\text {pro }}$ enzyme (Figure 4). 
The daunorubicin created one hydrogen bond and one $\mathrm{p}$-alkyl interaction with the catalytic dyad CYS145 and HIS41, respectively. The other five hydrogen bonds were formed by the hydroxyl $(-\mathrm{OH})$ groups of daunorubicin with HIS164, Arg188, Thr190, ASP187 and GIn192 as presented in Figure 4A. ABBV-744 formed the interaction network of six hydrogen bonds with Arg188, Thr190, GLU166, HIS163, GLN189 and GIn192 with the hydroxyl group $(-\mathrm{OH})$ of the compound, Figure 4B. The catalytic dyad CYS145, HYS41 and MET165 formed p-p stacking interaction and p-Sulphur interactions with ABBV-744. Onalespib formed an interaction network of total four hydrogen bonds with THR26, ARG188, THR 190 and GLN192. The catalytic dyad and MET165 interacted to Onalespib through p-p and p-sulphur interactions (Figure 4C).

Table 1: The best three compounds generated using XP docking with their corresponding docking scores and the interacting binding residues are presented. Catalytic dyad residues are shown in bold.

\begin{tabular}{|c|c|c|}
\hline Compounds & $\begin{array}{c}\text { Glide } \\
\text { GScore kcal/mol }\end{array}$ & Interacting binding residues \\
\hline Daunorubicin & -9.33 & $\begin{array}{l}\text { ASP187, ARG188, GLN189, THR190, ALA191, GLN192, MET49, TYR54, } \\
\text { HIS41, VAL142, CYS145, GLY143, HIS163, HIS164, MET165, GLU166, } \\
\text { PRO168, CYS44, VAL168 }\end{array}$ \\
\hline Onalespib & -8.21 & $\begin{array}{l}\text { VAL1 186, HIS41, CYS145, SER144, GLY143, ASN142, LEU27, THR26, } \\
\text { THR25, MET49, LEU50, ASP187, ARG188, GLN189, THR190, ALA191, } \\
\text { GLN192, PRO168, LEU167, GLU166, MET165, HIS164 }\end{array}$ \\
\hline ABBV-744 & -7.79 & $\begin{array}{l}\text { HIS41, MET49, CYS145, SER144, GLY143, ASN142, LEU141, PHE140, } \\
\text { HIS163, HIS164, HIE172, MET165, GLU166, LEU167, PRO168, ASP187, } \\
\text { ARG188, GLN189, THR190, GLN192, }\end{array}$ \\
\hline Inhibitor & -6.75 & $\begin{array}{l}\text { HIE172, PHE140, LEU141, ASN142, GLY143, SER144, CYY 145, GLN189, } \\
\text { ARG188, ASP187, HIS41, CYS44, MET49, TYR54, PRO168, LEU167, } \\
\text { GLU166, MET165, HIS164, HIS163, THR25, PHE181, SER46, GLU47, } \\
\text { GLY170 }\end{array}$ \\
\hline
\end{tabular}

Thus, it could be contemplated that these three compounds bound favorably to the binding site of $\mathrm{M}^{\text {pro }}$ through hydrogen bond, p-p stacking and p-alkyl interactions mainly generated by CYS145, HIS41, MET165, HIS163, GLU166, GLN 189, Arg188, Thr190 and GIn192 as key contributing active residues into the docked complexes.

\subsection{ADMET analysis}

Pharmacokinetic and toxicity features were predicted using QikProp module of Schrodinger for Daunorubicin, Onalespid, ABBV-744 and ak-13b-inhibitor. Outcomes of pharmacokinetic and toxicity study are illustrated in Table 2. The selected properties of the compounds are representatives of influence metabolism, cell permeation, bioavailability and toxicity.

Table 2: In-silico ADMET screening of the selected compounds. 


\begin{tabular}{ccccccccc}
\hline Compounds & ${ }^{\mathrm{a}}$ CNS & ${ }^{\mathrm{b}} \mathrm{QPlogKhsa}$ & ${ }^{\mathrm{c}}$ SASA & ${ }^{\mathrm{d}} \mathrm{QPlogPo} / \mathrm{w}$ & ${ }^{\mathrm{e}} \mathrm{QPlogS}$ & ${ }^{\mathrm{f}} \mathrm{QPlogBB}$ & $\begin{array}{c}\text { g\%Human } \\
\text { Oral } \\
\text { Absorption }\end{array}$ & $\begin{array}{c}\mathrm{h} \text { RuleOf } \\
\text { Five }\end{array}$ \\
\hline Daunorubicin & -2 & -0.32 & 716.74 & 0.49 & -2.30 & -1.88 & 9.34 & 3 \\
\hline $\begin{array}{l}\text { Onalespib } \\
\text { ABBV-744 }\end{array}$ & -2 & 0.58 & 763.23 & 3.03 & -3.92 & -0.3 & 74.42 & 0 \\
\hline $\begin{array}{l}\text { ak-13b } \\
\text { inhibitor }\end{array}$ & -2 & -0.07 & 960.42 & 3.11 & -5.15 & -2.91 & 33.40 & 3 \\
\hline
\end{tabular}

apredicted central nervous system activity from -2 (inactive) to +2 (active). ${ }^{b}$ Prediction of binding to human serum albumin (acceptable range: -1.5-1.5). ${ }^{\mathrm{C}}$ Total Solvent Accessible Surface Area: SASA (acceptable range: 300-1000). dPredicted octanol/water partition coefficient (acceptable range: -2-6.5). ${ }^{\text {ePredicted aqueous }}$

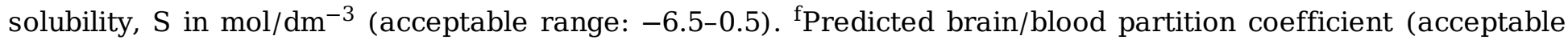
range: $-3.0-1.2)$. ${ }^{9}$ Predicted percentage human oral absorption ( $<25 \%$ is poor and $>80 \%$ is high). ${ }^{\mathrm{h}} \mathrm{Number}$ of violations of Lipinski's rule of five, Compounds that satisfy these rules are considered druglike (maximum 4).

The predicted central nervous system activity (CNS) of Daunorubicin, ABBV-744 and ak-13b inhibitor depicted as inactive whereas, Onalespib was presented as an active compound. The predicted human binding serum albumin (QPlogKhsa) of all compounds showed in the acceptable range. The estimated total solvent accessible surface area (SASA) of all three compounds and ak-13b inhibitor met the acceptable range: $300-1000$. Predicted octanol/water partition coefficient (QPlogPo/w) showed in the acceptable range from -2 to 6.5 for all the ligands. The predicted aqueous solubility (QPlogS) for Daunorubicin, Onalespib and ak-13b inhibitor were in the acceptable range of $-6.5-0.5$ whereas ABBV744 showed slightly low value. The predicted brain/blood partition coefficient (QPlogBB) for all these compounds showed in the acceptable ranges. The percentage human oral absorption for all the compounds met in the recommended range. Number of violations of Lipinski's rule of five for all the compounds satisfied this rule for all the studied ligands.

\subsection{Post-dynamics trajectories analysis}

The structural variations within the enzymes structure is correlated with their biological activities. Any alterations or interference on enzymes structural integrity might have a substantial impact on its activity [39]. The binding of inhibitors influence the mode of action of enzymes that are comprised in disease pathways, thus there is a requirement to estimate the structural dynamics and conformational changes associated with the inhibitory activity of these inhibitors [51].

In this section, 200 ns MD trajectories regarding the four complexes, namely, Daunorubicin-Mpro, Onalespib-M ${ }^{\text {pro }}, A B B V-744-M p r o$ and ak-13b inhibitor-M ${ }^{\text {pro }}$ as control model were analyzed. Different metrics and analysis were applied to investigate the stability and flexibility of the complexes as well as the contribution of the studied compounds upon binding in terms of binding free energies. The 2D chemical structure of all the ligands considered for MD simulations have been displayed in (Figure 1 and Scheme 1).

Scheme 1:2D chemical structure of top three hit compounds. (see Supplementary Files) 
The computation of a time variable with reference to an RMSD of $\mathrm{C}_{\mathrm{a}}$ atoms from generated trajectories was accomplished to investigate the consistency and efficiency of $\mathrm{M}^{\text {pro }}$ in complex with ak-13b inhibitor and along with the top three compounds, Figure 1 and scheme 1.

The perturbations in the RMSD values as denoted in plot (Figure 5A) throughout the simulation time disclosed the possible conformational deviances in the enzyme structure upon ligand binding. As Figure 5A revealed, all the complexes were stabilized and attained convergence after almost 50 ns of simulation run. ABBV-744-Mpro unveiled the lowest average RMSD of $2.45 \AA$, while Onalespid-Mpro and Daunorubicin-M ${ }^{\text {pro }}$ revealed average RMSD of $2.73 \AA$ and $2.76 \AA$ respectively. The ak-13b-inhibitor-Mpro unveils a perturbation of $2.85 \AA$ as indicated in the plot. This evaluation proposed that any further analyses performed on the produced trajectories of all complexes were reliable. The RMSD plots indicated that ABBV-744-Mpro, Onalespid-Mpro and Daunorubicin-M ${ }^{\text {pro }}$ complexes exhibit the lowest deviation of $\mathrm{C}^{\mathrm{a}}$ backbone atoms suggesting that stability of these three compounds inflicted higher constancy on $\mathrm{M}^{\text {pro }}$ enzyme as compared to the ak-13b inhibitor-M ${ }^{\text {pro }}$ complex.

To provide detailed insight into the structural fluctuation and flexibility of different regions of the amino acid residues of $\mathrm{M}^{\text {pro }}$ enzyme upon binding of the selected compounds, RMSF values for Ca atoms were calculated from trajectories generated over 200 ns of MD trajectories. Hence, ligand binding to the enzyme could be investigated in relation to the modification in flexibility in terms of RMSF values [52]. To discover the stringency and elasticity in $M^{\text {pro }}$ residues upon binding of chosen compounds, RMSF values for $\mathrm{C}^{\mathrm{a}}$ atoms were estimated from trajectories produced from $200 \mathrm{~ns}$ of MD simulations run. As shown in Figure 5B, ABBV-744-M ${ }^{\text {ro }}$ complex showed the least fluctuations in the amino acid residues with $7.56 \AA$. An average RMSF of $13.76 \AA$ and $15.09 \AA$ was spotted in complex Daunorubicin-M ${ }^{\text {pro }}$ and Onalespid$\mathrm{M}^{\text {pro }}$, correspondingly. The complex, ak-13b inhibitor-M $\mathrm{M}^{\text {ro }}$ disclosed an average of $14.11 \AA$ that is remarkably greater than $\mathrm{ABBV}-744-\mathrm{M}^{\mathrm{pro}}$ complex, signifying enhanced binding in comparison to the ak$13 \mathrm{~b}$ inhibitor-Mpro complex. This noteworthy decline might be coupled with structural inactivation that evidently confirmed as a result of significant binding of ABBV-744 compound in the active pocket of $M^{\text {pro }}$ enzyme. The reduced fluctuation of amino acid residues might have favored $M^{\text {pro }}$ enzyme inhibition through compound ABBV-744.

The radius of gyration $(\mathrm{Rg})$ parameter was assessed as the structural compactness index and its folding and unfolding behavior through the overall conformational variations in enzyme structure upon inhibitor binding. The mediocre values of $R_{\mathrm{g}}$ for $\mathrm{ABBV}-744-\mathrm{M}^{\text {pro }}$, Onalespid-M ${ }^{\text {pro }}$ and Daunorubicin-M ${ }^{\text {pro }}$ complexes were noted to be $41.55 \AA$, $41.87 \AA$ and $42.08 \AA$, respectively. Figure $5 \mathrm{C}$ plots disclosed extremely minor changes in the compactness of the three compounds. The compound ABBV-744 exhibited a lowest $R_{\mathrm{g}}$ in comparison with other two complexes and also with the control ak-13b inhibitor-Mpro complex (42.36 ̊), thus proposing improved compactness and enhanced binding with the $M^{\text {pro }}$ enzyme. All these patterns of conformational analysis are suggesting an improved stability, flexibility and compactness of compound ABBV-744 in complex the $M^{\text {pro }}$ enzyme. 
Solvent Access Surface Area (SASA) analysis was performed to define the activity of hydrophobic and hydrophilic amino acid residues and forces exposed to the solvent over $200 \mathrm{~ns}$ MD trajectories. The constant and accurate scheming of SASA is highly useful in the energetic evaluation of biological macromolecules [53]. The interfaces among the hydrophobic native contacts inside enzyme structure is a noteworthy intermolecular interaction that effect enzyme inhibition. Hydrophobic interaction constructed between the non-polar residues corroborate the stability of the enzyme structure in solution by sheltering the non-polar residues inside the hydrophobic core distant from an aqueous solution [54]. As displayed in Figure 5D, standard SASA values for all selected compounds have been measured during $200 \mathrm{~ns}$ MD trajectories. Average value of SASA for the compound ABBV-744-Mpro complex is $14230 \AA^{2}$ which was showed to the solvent system. Overall SASA values of $14303 \AA^{2}$ and $14426 \AA^{2}$ were prominent by Onalespid-M $\mathrm{M}^{\mathrm{pro}}$ and Daunorubicin-M $\mathrm{M}^{\text {pro }}$ complexes, individually. The differences in SASA values for all the complexes during the simulation period corresponds with the folding and unfolding of enzyme structure. The overall SASA values in the control complex was $14001 \AA^{2}$, slightly less than ABBV-744-Mpro complex. The SASA assessment perceived in compound ABBV-744 bound complex additionally validated that ABBV-744 compound has better exposure to solvent and consequently favored the improved inhibitory activity of compound ABBV-744 over other complexes.

\subsubsection{Hydrogen bond analysis}

For overall conformation and stability of enzyme structure, we have measured the intramolecular and intermolecular hydrogen bond analysis (Figure 6). This analysis gives extreme understanding into binding mechanism of enzyme-ligand with detailed consideration [55]. An average number of intramolecular hydrogen bonds in ABBV-744-M ${ }^{\text {pro }}$ complex was noted to be 136 as displayed in Figure 6A. In compound Onalespid and Daunorubicin, the intramolecular hydrogen bonds were observed to be 139 and 140 , respectively.

The number of intermolecular hydrogen bonds produced in the catalytic site of $\mathrm{M}^{\text {pro }}$ enzyme notable to be 9-10 in ABBV-744 bound $M^{\text {pro }}$ complex. However, number of these bonds are more in Daunorubicin$\mathrm{M}^{\mathrm{pro}}$ complex with 11-12 hydrogen bonds and less in Onalespid and ak-13b-inhibitor bound $\mathrm{M}^{\text {pro }}$ with 7-8 hydrogen bonds as presented in Figure 6B.

\subsubsection{Principal Component analysis}

To qualitatively probe the impact of inhibitor's binding on the predominant conformational motion of each residue, [56][57] the concerted motions in Daunorubicin-M $\mathrm{M}^{\mathrm{pro}}, \mathrm{ABBV}-744-\mathrm{M}^{\text {pro }}$ and Onalespid-M $\mathrm{M}^{\text {pro }}$ and ak-13b-inhibitor-M ${ }^{\text {pro }}$ complexes were studied using PC analysis based on the eigenvector. The scatter plots in Figure, essentially give a two-dimensional representation of the conformational changes occupied by the system. The gradual migration of the points in the PC1-PC2 scatter plots obtained using construction of eigenvectors, Figure 7A. PC1 collective motions extracted for the predominant eigenvectors of the using principal component in the studied complexes, Figure 7B. 
The scatter plots of the complexes in Figure, indicate the undergoing overall motions of the protein upon binding in terms of correlated and anti-correlated movements, Figure 7A and 7B.

It is evident in Figure 7A, the Onalespid, Daunorubicin and ak-13b-inhibitor with the trace covariance matric of $37.45 \AA^{2}, 37.67 \AA^{2}$ and $37.69 \AA^{2}$, imposed highly fluctuated anti-correlated effects as the negative values of 2D-scatter points into the protein, Figure 7B. Interestingly, in the case of ABBV-744 with the trace covariance matric of $37.64 \AA^{2}$, the prominent correlated motions were observed with the least fluctuations of the system upon ligand binding, Figure 7B. Thus, from the above observations Figure 7B, it was concluded that ABBV-744 induced least fluctuations into the binding site upon binding than the variants complexes.

\subsubsection{Mechanistic insights into binding affinity}

To understand the impact of inhibitors upon complexation in terms of their binding affinities, MM-GBSA binding free energy method were utilized to calculate the binding free energies and their components of the complexes, Table 3.

Table 3. Binding free energies and its components for the three hit compounds: $\mathrm{M}^{\text {pro }}$ and ak-ab-inhibitor: $\mathrm{M}^{\text {pro }}$ using MM-GBSA method. The energy components are in $\mathrm{kcal} \mathrm{mol}^{-1}$.

\begin{tabular}{|c|c|c|c|c|c|c|c|}
\hline Complex & $\mathrm{D} E_{\mathrm{vdw}}$ & $\mathrm{D} E_{\text {elec }}$ & $\mathrm{D} G_{\text {gas }}$ & $\mathrm{D} G_{\mathrm{polar}}$ & $\mathrm{D} G_{\text {nopolar }}$ & $\mathrm{D} G_{\text {solvation }}$ & $\mathrm{D} G_{\mathrm{bind}}$ \\
\hline Daunorubicin-M ${ }^{\text {pro }}$ & -49.65 & -4.39 & -54.05 & 22.48 & -5.09 & 17.39 & -36.65 \\
\hline Onalespib-M ${ }^{\text {pro }}$ & -43.55 & -18.55 & -62.10 & 29.42 & -4.45 & 24.97 & -37.13 \\
\hline ABBV-744-Mpro & -54.45 & -35.20 & -89.65 & 50.03 & -5.81 & 44.22 & -45.43 \\
\hline ak-3binhibitor-M ${ }^{\text {pro }}$ & -36.35 & -8.14 & -44.49 & 28.21 & -4.64 & 23.57 & -20.92 \\
\hline
\end{tabular}

As it is evident in Table 3, the total binding free energies $\left(\Delta G_{\text {bind }}\right)$ of Daunorubicin-M ${ }^{\text {pro }}$, Onalespib-Mro, ABBV-744-M ${ }^{\text {pro }}$ and ak-3binhibitor-M ${ }^{\text {pro }}$ were $-36.65 \mathrm{kcal} / \mathrm{mol},-37.13 \mathrm{kcal} \mathrm{mol}^{-1},-45.43 \mathrm{kcal} \mathrm{mol}^{-1}$ and $-20.92 \mathrm{kcal} \mathrm{mol}^{-1}$, respectively. Accordingly, among all the studied complexes, ABBV-744-M $\mathrm{Mro}^{\text {ro }}$ and Onalespib-M pro depicted the most favorable of $\Delta G_{\text {bind }}$ with lowest values of $-45.43 \mathrm{kcal} \mathrm{mol}^{-1}$ and -37.13 $\mathrm{kcal} \mathrm{mol}^{-1}$. At this point, it is interesting to address the key contributions that each binding component can impose to the total binding free energies.

It is evident that amongst the studied complexes, the $\Delta G_{\text {gas }}$ as the favorable contributing index into the total $\Delta G_{\text {bind }}$ has the lowest values for ABBV-744-M pro (-98.65 kcal mol-1) and Onalespib-M ${ }^{\text {pro }}(-62.10 \mathrm{kcal}$ $\mathrm{mol}^{-1}$ ) complexes. This observation implies the most favorable contribution values of $\Delta \mathrm{E}_{\mathrm{vdw}}$ and $\Delta \mathrm{E}_{\text {elec }}$ for ABBV-744-M pro (-54.45 kcal mol ${ }^{-1}$ and $\left.-35.20 \mathrm{kcal} \mathrm{mol}^{-1}\right)$ and Onalespib-M ${ }^{\text {pro }}\left(-43.55 \mathrm{kcal} \mathrm{mol}^{-1}\right.$ and $\left.-18.55 \mathrm{kcal} \mathrm{mol}^{-1}\right)$ into the total binding free energies.

Interestingly, this observation revealed another key contributing component of $D G_{\text {nonpolar }}$ for the complexes of ABBV-744-M ${ }^{\text {pro }}\left(-5.81 \mathrm{kcal} \mathrm{mol}^{-1}\right)$ and Onalespib-Mpro $\left(-4.45 \mathrm{kcal} \mathrm{mol}^{-1}\right)$ which leads to 
lowest $D G_{\text {bind }}$ values for both complexes. According to the obtained energy components results, it could be inferred that the $D E_{v d w}, D E_{\text {elec }}$ and $D G_{\text {nonpolar }}$ have the dominant contribution into the binding affinities for the selected complexes.

\subsubsection{Per-residue decomposition energy analysis}

The binding-free energy decomposition offers an immense understanding in account of enzyme-ligand complexes produced from the trajectories by MD simulations. To achieve this, we fragmented the total binding energies of complexes into each-residual involvement by per amino acid residue existing in the catalytic site of $\mathrm{M}^{\text {pro }}$ enzyme to provide comprehensive identification of key contributing residues upon ligand binding as depicted in Figure 8. The interactions between catalytic site electro-negative and electro-positive residues develops ligand binding and its stabilization at the target enzyme. This creates an improved intermolecular binding that surges the binding affinity of the ligand in the active site. Active site residue Met165 in ABBV-744 contributed with the lowest $\Delta G_{\text {bind }}$ with $-4.22 \mathrm{kcal} \mathrm{mol}^{-1}$ however this residue has contributed with notably less $\Delta G_{\text {bind }}$ of $-2.50 \mathrm{kcal} \mathrm{mol}^{-1}$ and $-2.45 \mathrm{kcal} \mathrm{mol}^{-1}$ in Onalespid and Daunorubicin bound complexes. The $\Delta G_{\text {bind }}$ of another participating residues Gln 189 was also observed to be lowest in ABBV-744 bound $\mathrm{M}^{\text {pro }}$ complex with $-3.94 \mathrm{kcal} \mathrm{mol}^{-1}$ however, it is slightly less in Onalespid-M pro complex with value of $-3.45 \mathrm{kcal} \mathrm{mol}^{-1}$ and $-2.48 \mathrm{kcal} \mathrm{mol}^{-1}$ in Daunorubicin-M $\mathrm{M}^{\text {pro }}$ complex. Gln192 significantly contributed in the binding of ABBV-744 compound with $\Delta G_{\text {bind }}$ value of $-3.00 \mathrm{kcal}$ $\mathrm{mol}^{-1}$ and observed a very minor difference with $-2.99 \mathrm{kcal} \mathrm{mol}^{-1} \Delta G_{\text {bind }}$ in Onalespid bound $\mathrm{M}^{\text {pro }}$ but showed a lesser $\Delta G_{\text {bind }}$ of $-1.50 \mathrm{kcal} \mathrm{mol}^{-1}$ in Daunorubicin compound.

Glu47, Phe140, Leu141, Asn142, Gly143, Ser144 and Glu166 are the other active site amino acid residues which favored with the negative binding of ABBV-744 compound with $\Delta G_{\text {bind }}$ values of $-0.48 \mathrm{kcal} \mathrm{mol}^{-1}$, $-0.39 \mathrm{kcal} \mathrm{mol}^{-1},-0.52 \mathrm{kcal} \mathrm{mol}^{-1},-0.50 \mathrm{kcal} \mathrm{mol}^{-1},-0.14 \mathrm{kcal} \mathrm{mol}^{-1},-0.41 \mathrm{kcal} \mathrm{mol}^{-1}$ and $-1.50 \mathrm{kcal} \mathrm{mol}^{-1}$ respectively, however, these residues contributed with positive energies in Onalespid and Daunorubicin bound $\mathrm{M}^{\text {pro }}$ complexes. The catalytic dyad residues, His41 and Cys 145 showed the lowest $\Delta G_{\text {bind }}$ value of $-1.64 \mathrm{kcal} \mathrm{mol}^{-1}$ and $-0.61 \mathrm{kcalmol}^{-1}$ in control inhibitor ak-3b-inhibitor bound $\mathrm{M}^{\text {pro }}$, although these residues showed less binding energies in ABBV-744, Onalespud and Daunorubicin bound $M^{\text {pro }}$ complexes. Thus, this assessment discloses that similar binding residues contributing into the overall binding energies of the ABBV-744-M ${ }^{\text {pro }}$ complex indicative of ABBV-744 compound binds significantly to $\mathrm{M}^{\text {pro }}$ enzyme.

\section{Conclusions}

The necessity to control alarming COVID-19 pandemic made us to rationalize potential lead compounds that could be considered in clinical trials. Despite major investigations in the design and development of specific drugs or vaccines, not much proven to be effective against COVID-19. This challenge motivated us to explore the drug designing approaches that could serve informative to combat this disease. In this 
report, we have performed 3Dstructure-based pharmacophore modeling followed by virtual screeningbased 3D-pharmacophore hypotheses of 75 compounds as potential antiviral agents retrieved from PubChem. Molecular docking workflow using HTVS, SP and XP protocols were used to generate the best hits and their corresponding docked poses. The Six best compounds generated based on their lowest docking binding affinities using XP were considered for ADMET prediction-based physicochemical and pharmacokinetic descriptors and MD simulations analysis. MD simulations approach revealed the two highly selective compounds namely, ABBV-744 and Onalespid possessed significant binding affinity and presumably inhibition of SARS-CoV-2 $\mathrm{M}^{\text {pro }}$ enzyme. Based on our overall observations, compounds ABBV-744 and Onalespid could be recommended as potential lead for the therapeutic of COVID-19 patients.

\section{Declarations}

\section{Acknowledgments}

SK and ZF would like to acknowledge CHPC server based in Cape Town, South Africa and SK would also like to thank University of the Witwatersrand URC postdoctoral fellowship and Faculty of Health Sciences Research Committee (FRC, Grant no: 001254846410151211050000000000000000 5254) for conducting this research.

\section{Declaration of Competing Interest}

The authors declare that they have no known competing financial interests or personal relationships that could have appeared to influence the work reported in this paper.

\section{Supplementary data}

Supplementary material related to this article can be found, in the online version.

\section{References}

[1] H.A. Rothan, S.N. Byrareddy, The epidemiology and pathogenesis of coronavirus disease (COVID19) outbreak, J. Autoimmun. 109 (2020). https://doi.org/10.1016/j.jaut.2020.102433.

[2] S.F. Ahmed, A.A. Quadeer, M.R. McKay, Preliminary identification of potential vaccine targets for the COVID-19 Coronavirus (SARS-CoV-2) Based on SARS-CoV Immunological Studies, Viruses. 12 (2020). https://doi.org/10.3390/v12030254.

[3] M.T. ul Qamar, S.M. Alqahtani, M.A. Alamri, L.-L. Chen, Structural basis of SARS-CoV-2 3CLpro and anti-COVID-19 drug discovery from medicinal plants, J. Pharm. Anal. (2020). https://doi.org/10.1016/j.jpha.2020.03.009. 
[4] J. Liu, X. Zheng, Q. Tong, W. Li, B. Wang, K. Sutter, M. Trilling, M. Lu, U. Dittmer, D. Yang, Overlapping and discrete aspects of the pathology and pathogenesis of the emerging human pathogenic coronaviruses SARS-CoV, MERS-CoV, and 2019-nCoV, J. Med. Virol. 92 (2020) 491-494. https://doi.org/10.1002/jmv.25709.

[5] Y.F. Tu, C.S. Chien, A.A. Yarmishyn, Y.Y. Lin, Y.H. Luo, Y.T. Lin, W.Y. Lai, D.M. Yang, S.J. Chou, Y.P. Yang, M.L. Wang, S.H. Chiou, A review of sars-cov-2 and the ongoing clinical trials, Int. J. Mol. Sci. 21 (2020). https://doi.org/10.3390/ijms21072657.

[6] A.A. Rabaan, S.H. Al-Ahmed, S. Haque, R. Sah, R. Tiwari, Y.S. Malik, K. Dhama, M.I. Yatoo, D.K. Bonilla-Aldana, A.J. Rodriguez-Morales, SARS-CoV-2, SARS-CoV, and MERS-COV: A comparative overview, Le Infez. Med. 28 (2020) 174-184.

[7] B. Shanmugaraj, K. Siriwattananon, K. Wangkanont, W. Phoolcharoen, Perspectives on monoclonal antibody therapy as potential therapeutic intervention for Coronavirus disease-19 (COVID-19), Asian Pacific J. Allergy Immunol. 38 (2020) 10-18. https://doi.org/10.12932/AP-200220-0773.

[8] C. Wu, Y. Liu, Y. Yang, P. Zhang, W. Zhong, Y. Wang, Q. Wang, Y. Xu, M. Li, X. Li, M. Zheng, L. Chen, H. $\mathrm{Li}$, Analysis of therapeutic targets for SARS-CoV-2 and discovery of potential drugs by computational methods, Acta Pharm. Sin. B. (2020). https://doi.org/10.1016/j.apsb.2020.02.008.

[9] Z. Jin, X. Du, Y. Xu, Y. Deng, M. Liu, Y. Zhao, B. Zhang, X. Li, L. Zhang, C. Peng, Y. Duan, J. Yu, L. Wang, K. Yang, F. Liu, R. Jiang, X. Yang, T. You, X. Liu, X. Yang, F. Bai, H. Liu, X. Liu, L.W. Guddat, W. Xu, G. Xiao, C. Qin, Z. Shi, H. Jiang, Z. Rao, H. Yang, Structure of Mpro from COVID-19 virus and discovery of its inhibitors, Nature. (2020). https://doi.org/10.1038/s41586-020-2223-y.

[10] L. Zhang, D. Lin, X. Sun, U. Curth, C. Drosten, L. Sauerhering, S. Becker, K. Rox, R. Hilgenfeld, Crystal structure of SARS-CoV-2 main protease provides a basis for design of improved a-ketoamide inhibitors, Science. 368 (2020) 409-412. https://doi.org/10.1126/science.abb3405.

[11] J. Tan, S. George, Y. Kusov, M. Perbandt, S. Anemuller, J.R. Mesters, H. Norder, B. Coutard, C. Lacroix, P. Leyssen, J. Neyts, R. Hilgenfeld, 3C Protease of Enterovirus 68: Structure-Based Design of Michael Acceptor Inhibitors and Their Broad-Spectrum Antiviral Effects against Picornaviruses, J. Virol. 87 (2013) 4339-4351. https://doi.org/10.1128/jvi.01123-12.

[12] P.S. Dragovich, R. Zhou, D.J. Skalitzky, S.A. Fuhrman, A.K. Patick, C.E. Ford, J.W. Meador, S.T. Worland, Solid-phase synthesis of irreversible human rhinovirus $3 \mathrm{C}$ protease inhibitors. Part 1: Optimization of tripeptides incorporating N-terminal amides, in: Bioorganic Med. Chem., Bioorg Med Chem, 1999: pp. 589-598. https://doi.org/10.1016/S0968-0896(99)00005-X.

[13] D.E. Gordon, G.M. Jang, M. Bouhaddou, J. Xu, K. Obernier, K.M. White, M.J. O'meara, V. V Rezelj, J.Z. Guo, D.L. Swaney, T.A. Tummino, R. Huettenhain, R.M. Kaake, A.L. Richards, B. Tutuncuoglu, H. Foussard, J. Batra, K. Haas, M. Modak, M. Kim, P. Haas, B.J. Polacco, H. Braberg, J.M. Fabius, M. Eckhardt, M. 
Soucheray, M.J. Bennett, M. Cakir, M.J. Mcgregor, Q. Li, B. Meyer, F. Roesch, T. Vallet, A. Mac Kain, L. Miorin, E. Moreno, Z. Zar, C. Naing, Y. Zhou, S. Peng, Y. Shi, Z. Zhang, W. Shen, I.T. Kirby, J.E. Melnyk, J.S. Chorba, K. Lou, S.A. Dai, I. Barrio-Hernandez, D. Memon, C. Hernandez-Armenta, J. Lyu, C.J.P. Mathy, T. Perica, K.B. Pilla, S.J. Ganesan, D.J. Saltzberg, R. Rakesh, X. Liu, S.B. Rosenthal, L. Calviello, S. Venkataramanan, J. Liboy-Lugo, Y. Lin, X.-P. Huang, Y. Liu, S.A. Wankowicz, M. Bohn, M. Safari, F.S. Ugur, C. Koh, N. Sadat Savar, Q.D. Tran, D. Shengjuler, S.J. Fletcher, M.C. O’neal, Y. Cai, J.C.J. Chang, D.J. Broadhurst, S. Klippsten, P.P. Sharp, N.A. Wenzell, D. Kuzuoglu, H.-Y. Wang, R. Trenker, J.M. Young, D.A. Cavero, J. Hiatt, T.L. Roth, U. Rathore, A. Subramanian, J. Noack, M. Hubert, R.M. Stroud, A.D. Frankel, O.S. Rosenberg, K.A. Verba, D.A. Agard, M. Ott, M. Emerman, N. Jura, M. Von Zastrow, E. Verdin, A. Ashworth, O. Schwartz, C. D’enfert, S. Mukherjee, M. Jacobson, H.S. Malik, D.G. Fujimori, T. Ideker, C.S. Craik, S.N. Floor, J.S. Fraser, J.D. Gross, A. Sali, B.L. Roth, D. Ruggero, J. Taunton, T. Kortemme, P. Beltrao, M. Vignuzzi, A. García-Sastre, K.M. Shokat, B.K. Shoichet, N.J. Krogan, A SARS-CoV-2 protein interaction map reveals targets for drug repurposing A SARS-CoV-2 protein interaction map reveals targets for drug repurposing. Nature, Nature. (2020). https://doi.org/10.1038/s41586-020-2286-9.

[14] Protein Preparation Wizard | Schrödinger, (n.d.). https://www.schrodinger.com/protein-preparationwizard (accessed April 17, 2020).

[15] E. Harder, W. Damm, J. Maple, C. Wu, M. Reboul, J.Y. Xiang, L. Wang, D. Lupyan, M.K. Dahlgren, J.L. Knight, J.W. Kaus, D.S. Cerutti, G. Krilov, W.L. Jorgensen, R. Abel, R.A. Friesner, OPLS3: A Force Field Providing Broad Coverage of Drug-like Small Molecules and Proteins, J. Chem. Theory Comput. 12 (2016) 281-296. https://doi.org/10.1021/acs.jctc.5b00864.

[16] D. Shivakumar, J. Williams, Y. Wu, W. Damm, J. Shelley, W. Sherman, Prediction of absolute solvation free energies using molecular dynamics free energy perturbation and the opls force field, J. Chem. Theory Comput. 6 (2010) 1509-1519. https://doi.org/10.1021/ct900587b.

[17] J.C. Shelley, A. Cholleti, L.L. Frye, J.R. Greenwood, M.R. Timlin, M. Uchimaya, Epik: A software program for pKa prediction and protonation state generation for drug-like molecules, J. Comput. Aided. Mol. Des. 21 (2007) 681-691. https://doi.org/10.1007/s10822-007-9133-z.

[18] S.L. Dixon, A.M. Smondyrev, E.H. Knoll, S.N. Rao, D.E. Shaw, R.A. Friesner, PHASE: A new engine for pharmacophore perception, 3D QSAR model development, and 3D database screening: 1. Methodology and preliminary results, J. Comput. Aided. Mol. Des. 20 (2006) 647-671.

https://doi.org/10.1007/s10822-006-9087-6.

[19] N.K. Salam, R. Nuti, W. Sherman, Novel method for generating structure-based pharmacophores using energetic analysis, J. Chem. Inf. Model. 49 (2009) 2356-2368. https://doi.org/10.1021/ci900212v.

[20] T.A. Halgren, R.B. Murphy, R.A. Friesner, H.S. Beard, L.L. Frye, W.T. Pollard, J.L. Banks, Glide: A New Approach for Rapid, Accurate Docking and Scoring. 2. Enrichment Factors in Database Screening, J. Med. Chem. 47 (2004) 1750-1759. https://doi.org/10.1021/jm030644s. 
[21] C.A. Lipinski, Lead- and drug-like compounds: The rule-of-five revolution, Drug Discov. Today Technol. 1 (2004) 337-341. https://doi.org/10.1016/j.ddtec.2004.11.007.

[22] C.A. Lipinski, F. Lombardo, B.W. Dominy, P.J. Feeney, Experimental and computational approaches to estimate solubility and permeability in drug discovery and development settings, Adv. Drug Deliv. Rev. 46 (2001) 3-26. https://doi.org/10.1016/S0169-409X(00)00129-0.

[23] M. Karplus, J.A. McCammon, Molecular dynamics simulations of biomolecules, Nat. Struct. Biol. 9 (2002) 646-652. https://doi.org/10.1038/nsb0902-646.

[24] J.A. McCammon, B.R. Gelin, M. Karplus, Dynamics of folded proteins, Nature. 267 (1977) 585-590. https://doi.org/10.1038/267585a0.

[25] M. Arnittali, A.N. Rissanou, V. Harmandaris, Structure of Biomolecules Through Molecular Dynamics Simulations, in: Procedia Comput. Sci., Elsevier B.V., 2019: pp. 69-78.

https://doi.org/10.1016/j.procs.2019.08.181.

[26] T.S. Lee, D.S. Cerutti, D. Mermelstein, C. Lin, S. Legrand, T.J. Giese, A. Roitberg, D.A. Case, R.C. Walker, D.M. York, GPU-Accelerated Molecular Dynamics and Free Energy Methods in Amber18: Performance Enhancements and New Features, J. Chem. Inf. Model. 58 (2018) 2043-2050. https://doi.org/10.1021/acs.jcim.8b00462.

[27] A.W. Götz, M.J. Williamson, D. Xu, D. Poole, S. Le Grand, R.C. Walker, Routine microsecond molecular dynamics simulations with AMBER on GPUs. 1. generalized born, J. Chem. Theory Comput. 8 (2012) 1542-1555. https://doi.org/10.1021/ct200909j.

[28] V. Hornak, R. Abel, A. Okur, B. Strockbine, A. Roitberg, C. Simmerling, Comparison of multiple amber force fields and development of improved protein backbone parameters, Proteins Struct. Funct. Genet. 65 (2006) 712-725. https://doi.org/10.1002/prot.21123.

[29] J. Wang, R.M. Wolf, J.W. Caldwell, P.A. Kollman, D.A. Case, Development and testing of a general Amber force field, J. Comput. Chem. 25 (2004) 1157-1174. https://doi.org/10.1002/jcc.20035.

[30] J. Wang, W. Wang, P.A. Kollman, D.A. Case, Automatic atom type and bond type perception in molecular mechanical calculations, J. Mol. Graph. Model. 25 (2006) 247-260.

https://doi.org/10.1016/j.jmgm.2005.12.005.

[31] M.F. Harrach, B. Drossel, Structure and dynamics of TIP3P, TIP4P, and TIP5P water near smooth and atomistic walls of different hydroaffinity, J. Chem. Phys. 140 (2014) 174501. https://doi.org/10.1063/1.4872239.

[32] M.J. Harvey, G. De Fabritiis, An implementation of the smooth particle mesh Ewald method on GPU hardware, J. Chem. Theory Comput. (2009). https://doi.org/10.1021/ct900275y. 
[33] J.P. Ryckaert, G. Ciccotti, H.J.C. Berendsen, Numerical integration of the cartesian equations of motion of a system with constraints: molecular dynamics of n-alkanes, J. Comput. Phys. (1977). https://doi.org/10.1016/0021-9991(77)90098-5.

[34] Y. Lin, D. Pan, J. Li, L. Zhang, X. Shao, Application of Berendsen barostat in dissipative particle dynamics for nonequilibrium dynamic simulation, J. Chem. Phys. 146 (2017).

https://doi.org/10.1063/1.4978807.

[35] D.R. Roe, T.E. Cheatham, PTRAJ and CPPTRAJ: Software for processing and analysis of molecular dynamics trajectory data, J. Chem. Theory Comput. 9 (2013) 3084-3095. https://doi.org/10.1021/ct400341p.

[36] E. Seifert, OriginPro 9.1: Scientific data analysis and graphing software - Software review, J. Chem. Inf. Model. 54 (2014) 1552. https://doi.org/10.1021/ci500161d.

[37] H. Wan, J.P. Hu, X.H. Tian, S. Chang, Molecular dynamics simulations of wild type and mutants of human complement receptor 2 complexed with C3d, Phys. Chem. Chem. Phys. 15 (2013) 1241-1251. https://doi.org/10.1039/c2cp41388d.

[38] S. Chang, J.P. Hu, P.Y. Lin, X. Jiao, X.H. Tian, Substrate recognition and transport behavior analyses of amino acid antiporter with coarse-grained models, Mol. Biosyst. 6 (2010) 2430-2438. https://doi.org/10.1039/c005266c.

[39] Z. Fakhar, T. Govender, G.E.M. Maguire, G. Lamichhane, R.C. Walker, H.G. Kruger, B. Honarparvar, Differential flap dynamics in I,d-transpeptidase2 from mycobacterium tuberculosis revealed by molecular dynamics, Mol. Biosyst. 13 (2017) 1223-1234. https://doi.org/10.1039/c7mb00110j.

[40] C.C. David, D.J. Jacobs, Principal component analysis: A method for determining the essential dynamics of proteins, Methods Mol. Biol. 1084 (2014) 193-226. https://doi.org/10.1007/978-1-62703658-0_11.

[41] R.M. Levy, A.R. Srinivasan, W.K. Olson, J.A. McCammon, Quasi-harmonic method for studying very low frequency modes in proteins, Biopolymers. 23 (1984) 1099-1112.

https://doi.org/10.1002/bip.360230610.

[42] J. Chen, J. Wang, W. Zhu, Binding modes of three inhibitors 8CA, F8A and I4A to A-FABP studied based on molecular dynamics simulation, PLoS One. 9 (2014).

https://doi.org/10.1371/journal.pone.0099862.

[43] M. Laberge, T. Yonetani, Molecular dynamics simulations of hemoglobin a in different states and bound to DPG: Effector-linked perturbation of tertiary conformations and $\mathrm{HbA}$ concerted dynamics, Biophys. J. 94 (2008) 2737-2751. https://doi.org/10.1529/biophysj.107.114942. 
[44] T. Ichiye, M. Karplus, Collective motions in proteins: A covariance analysis of atomic fluctuations in molecular dynamics and normal mode simulations, Proteins Struct. Funct. Bioinforma. 11 (1991) 205217. https://doi.org/10.1002/prot.340110305.

[45] W. Humphrey, A. Dalke, K. Schulten, VMD: Visual molecular dynamics, J. Mol. Graph. 14 (1996) 3338. https://doi.org/10.1016/0263-7855(96)00018-5.

[46] E. Wang, H. Sun, J. Wang, Z. Wang, H. Liu, J.Z.H. Zhang, T. Hou, End-Point Binding Free Energy Calculation with MM/PBSA and MM/GBSA: Strategies and Applications in Drug Design, Chem. Rev. 119 (2019) 9478-9508. https://doi.org/10.1021/acs.chemrev.9b00055.

[47] H. Gohlke, C. Kiel, D.A. Case, Insights into protein-protein binding by binding free energy calculation and free energy decomposition for the Ras-Raf and Ras-RalGDS complexes., J. Mol. Biol. 330 (2003) 891-913. https://doi.org/10.1016/S0022-2836(03)00610-7.

[48] J. Weiser, P.S. Shenkin, W.C. Still, Approximate atomic surfaces from linear combinations of pairwise overlaps (LCPO), J. Comput. Chem. 20 (1999) 217-230. https://doi.org/10.1002/(SICI)1096987X(19990130)20:2<217::AID-JCC4>3.0.CO;2-A.

[49] A.R. Leach, V.J. Gillet, R.A. Lewis, R. Taylor, Three-dimensional pharmacophore methods in drug discovery, J. Med. Chem. 53 (2010) 539-558. https://doi.org/10.1021/jm900817u.

[50] R.A. Friesner, R.B. Murphy, M.P. Repasky, L.L. Frye, J.R. Greenwood, T.A. Halgren, P.C. Sanschagrin, D.T. Mainz, Extra precision glide: Docking and scoring incorporating a model of hydrophobic enclosure for protein-ligand complexes, J. Med. Chem. 49 (2006) 6177-6196. https://doi.org/10.1021/jm051256o.

[51] S. Khan, I. Bjij, M.E.S. Soliman, Selective Covalent Inhibition of "Allosteric Cys121" Distort the Binding of PTP1B Enzyme: A Novel Therapeutic Approach for Cancer Treatment, Cell Biochem. Biophys. 77 (2019) 203-211. https://doi.org/10.1007/s12013-019-00882-5.

[52] S. Khan, I. Bjij, R.M. Betz, M.E.S. Soliman, Reversible versus irreversible inhibition modes of ERK2: A comparative analysis for ERK2 protein kinase in cancer therapy, Future Med. Chem. 10 (2018) 10031015. https://doi.org/10.4155/fmc-2017-0275.

[53] A. Fornili, F. Autore, N. Chakroun, P. Martinez, F. Fraternali, Protein-water interactions in MD simulations: POPS/POPSCOMP solvent accessibility analysis, solvation forces and hydration sites, Methods Mol. Biol. 819 (2012) 375-392. https://doi.org/10.1007/978-1-61779-465-0_23.

[54] H. Chen, A.Z. Panagiotopoulos, Molecular modeling of surfactant micellization using solventaccessible surface area, Langmuir. 35 (2019) 2443-2450.

https://doi.org/10.1021/acs.langmuir.8b03440.

[55] T. Mohammad, S. Batra, R. Dahiya, M.H. Baig, I.A. Rather, J.J. Dong, I. Hassan, Identification of highaffinity inhibitors of cyclin-dependent kinase 2 towards anticancer therapy, Molecules. 24 (2019). 
https://doi.org/10.3390/molecules24244589.

[56] H.M. Kumalo, S. Bhakat, M.E. Soliman, Investigation of flap flexibility of $\beta$-secretase using molecular dynamic simulations, J. Biomol. Struct. Dyn. 34 (2016) 1008-1019. https://doi.org/10.1080/07391102.2015.1064831.

[57] B. Nizami, D. Sydow, G. Wolber, B. Honarparvar, Molecular insight on the binding of NNRTI to K103N mutated HIV-1 RT: Molecular dynamics simulations and dynamic pharmacophore analysis, Mol. Biosyst. 12 (2016) 3385-3395. https://doi.org/10.1039/c6mb00428h.

\section{Figures}

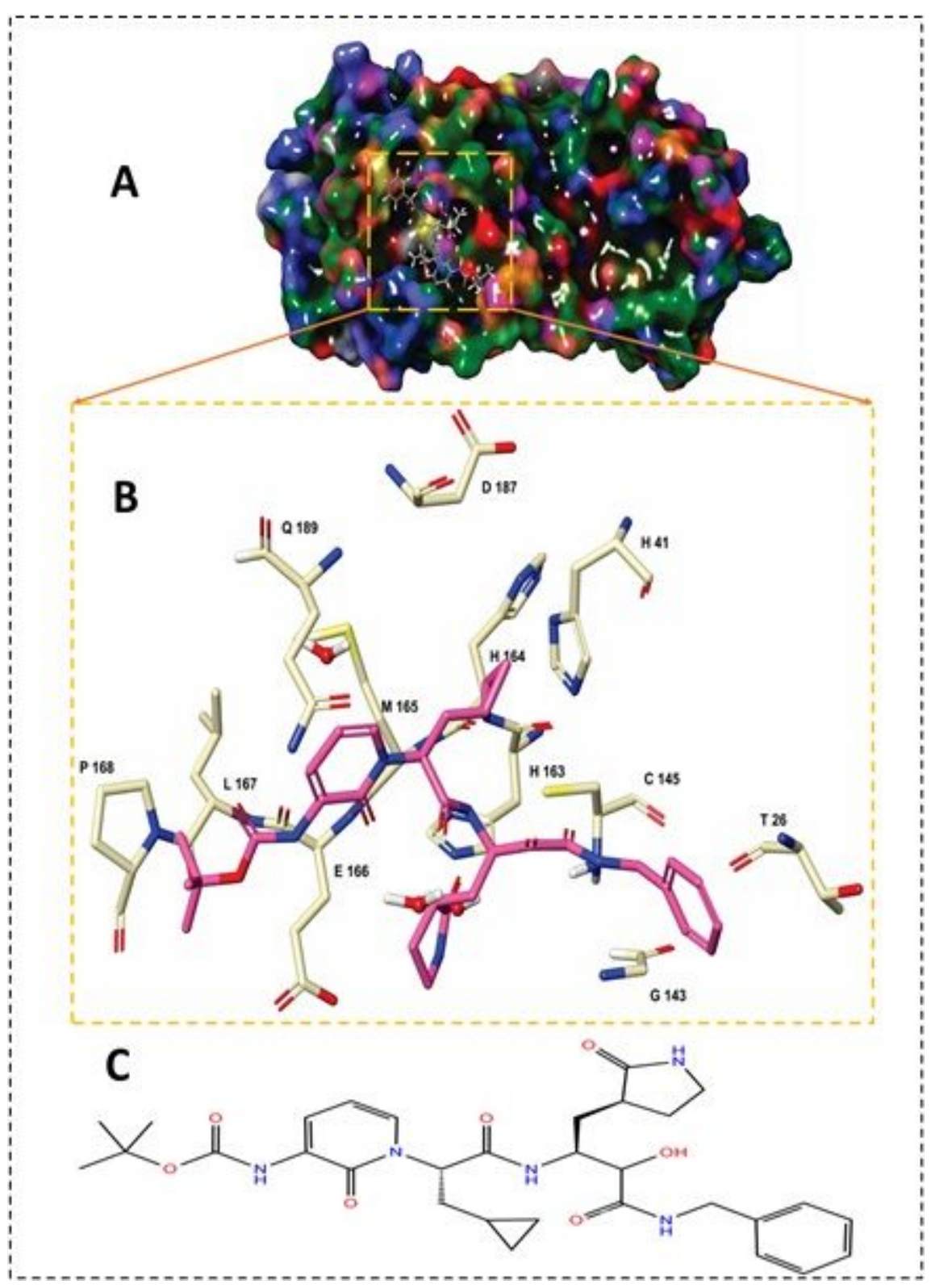

Figure 1 


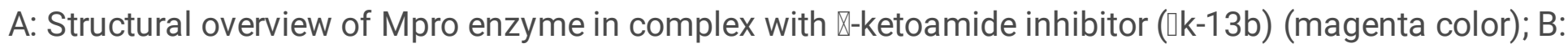

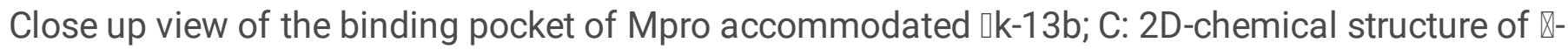
ketoamide inhibitor ( $(\mathrm{k}-13 \mathrm{~b})$

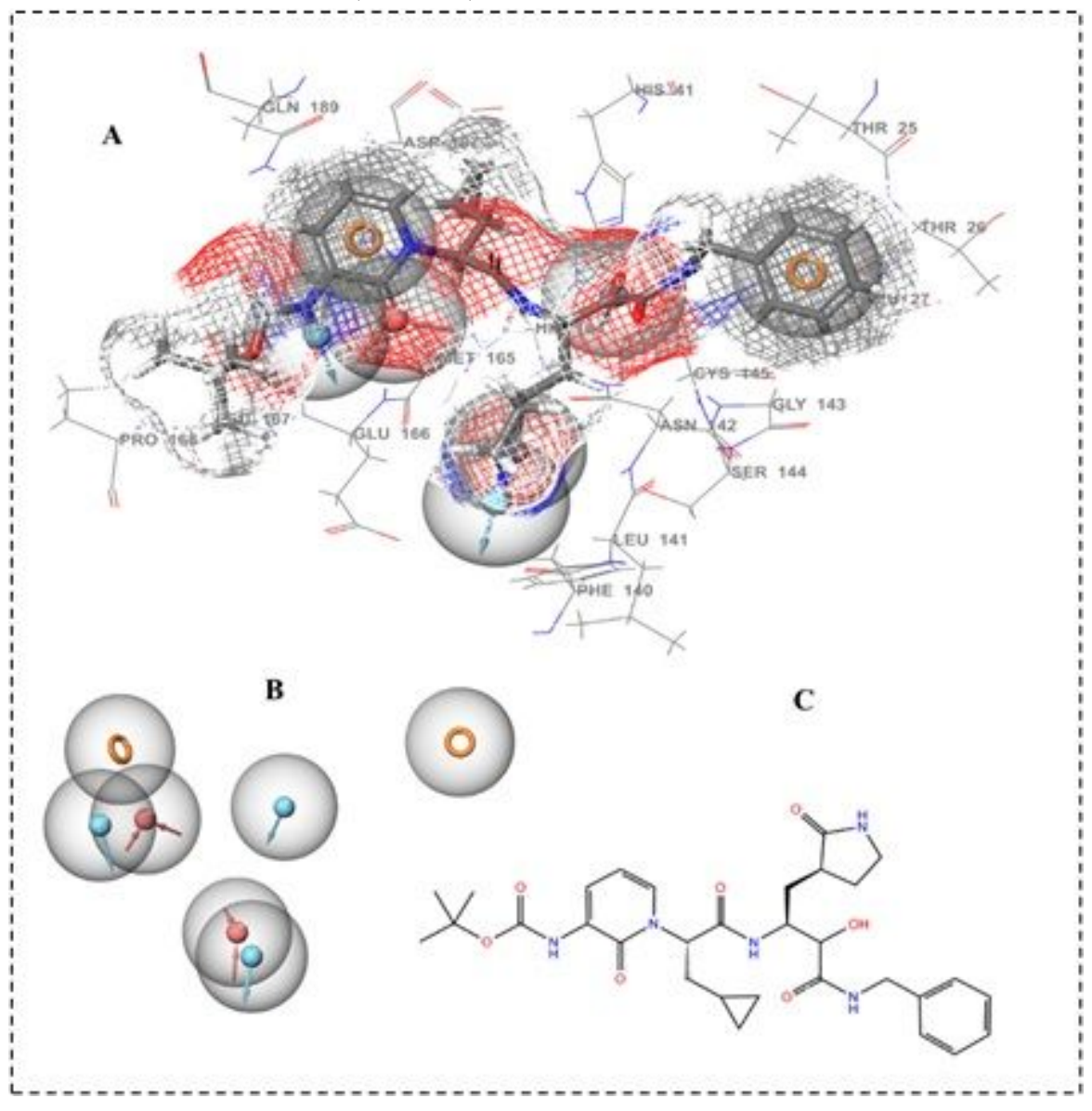

Figure 2

(A) 3D features of structure-based pharmacophore features of 3b-inhibitor in the complex interacting with Mpro binding site. (B) The seven generated pharmacophore features in Red arrow: Hydrogen bond acceptor, blue arrows: Hydrogen bond donor, orange: aromatic ring. (C) 2D chemical structure of the inhibitor. 


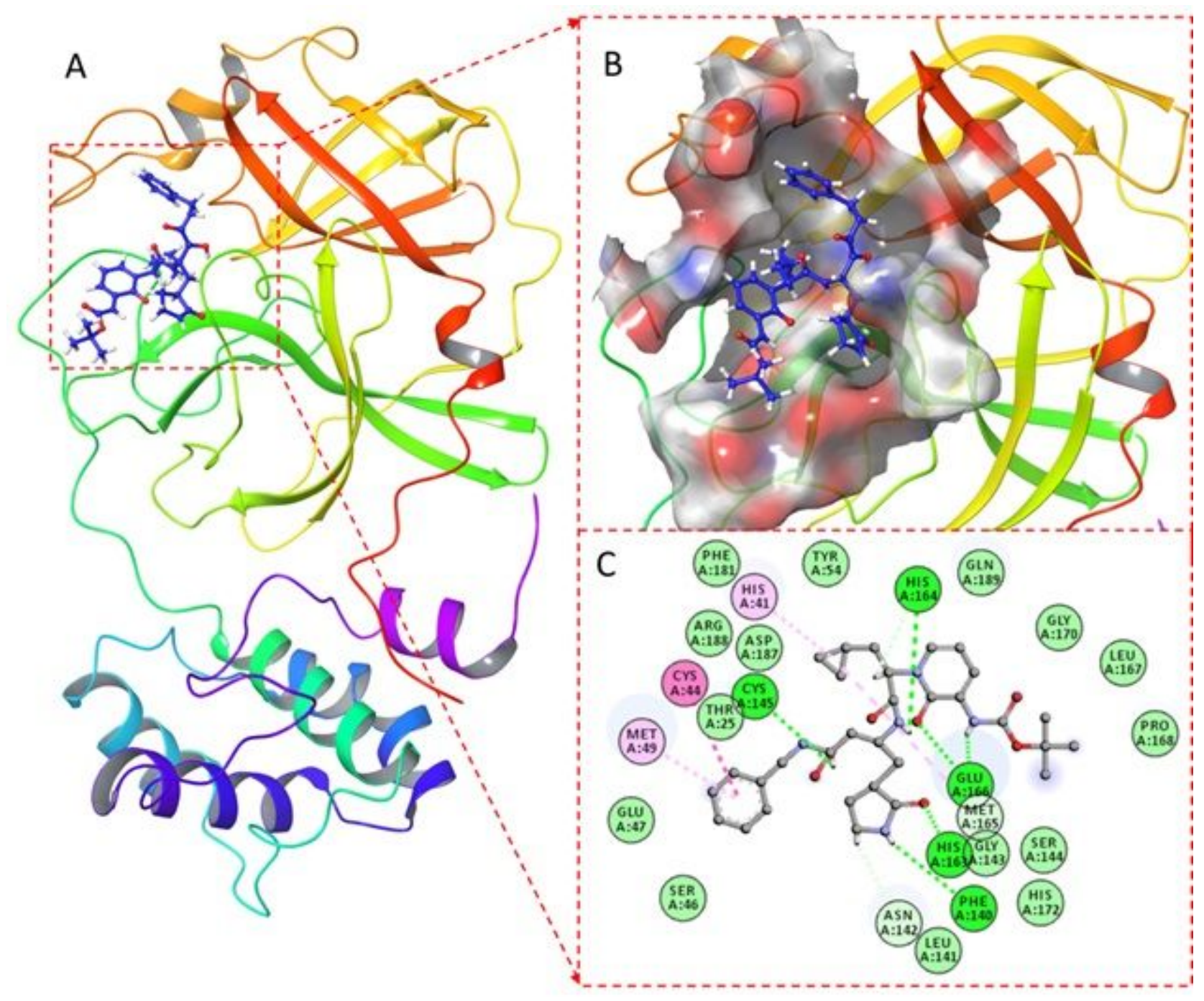

Figure 3

A. $\square$ k-3b-inhibitor in complex with Mpro enzyme, B. Surface view colored by charge showing the catalytic pocket of the Mpro enzyme; C. 2D representation of the interaction map of docked inhibitor in complex with Mpro. 


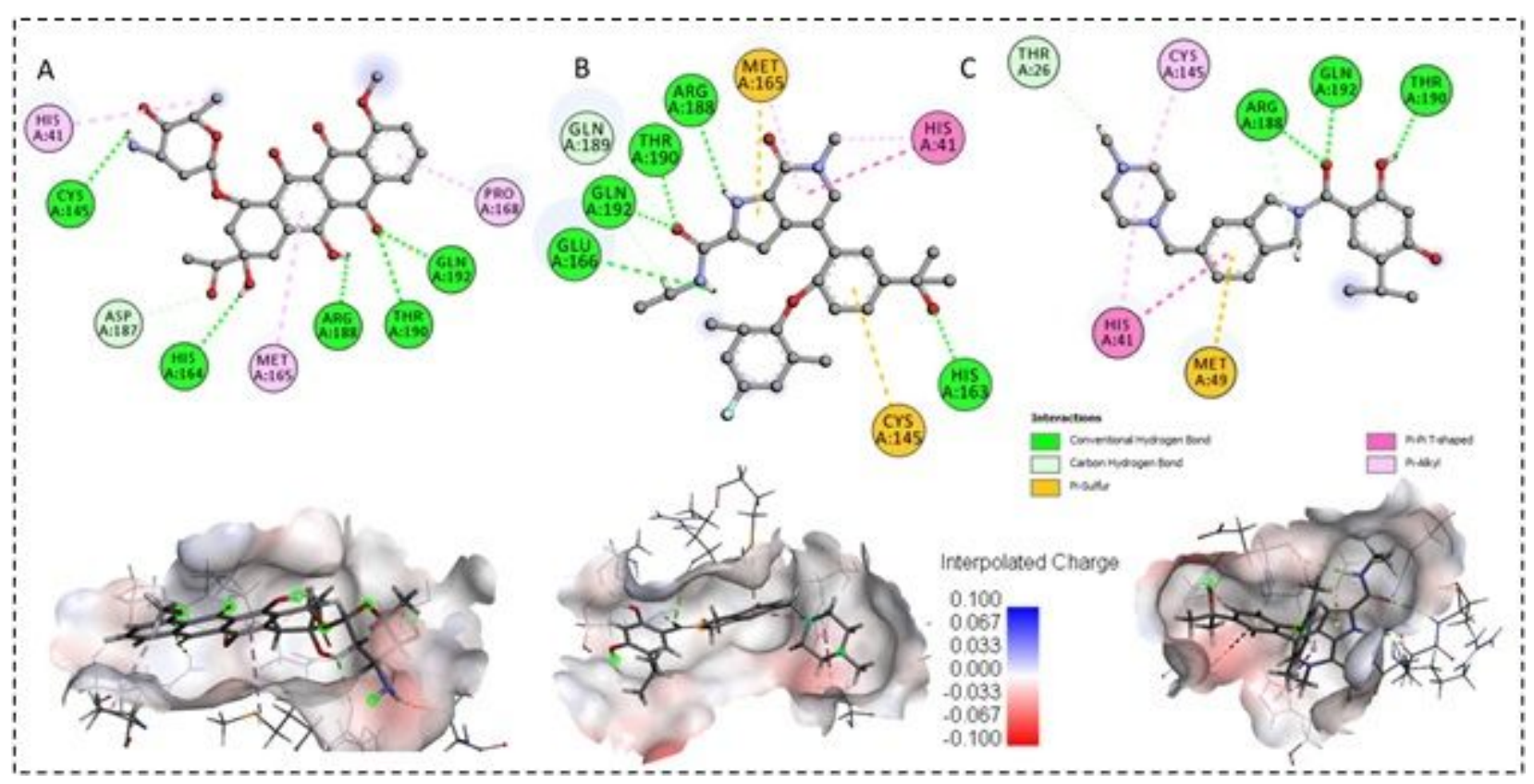

Figure 4

Docked poses of Mpro enzyme with the proposed inhibitors. 2D plots and binding interactions of Mpro enzyme with compounds (A) Daunorubicin (B) ABBV-744 and (C) Onalespib. Lower panels are representing the surface view of conserved substrate -binding pocket of Mpro in complexed with Daunorubicin, ABBV-744 and Onalespid, respectively (left to right). 

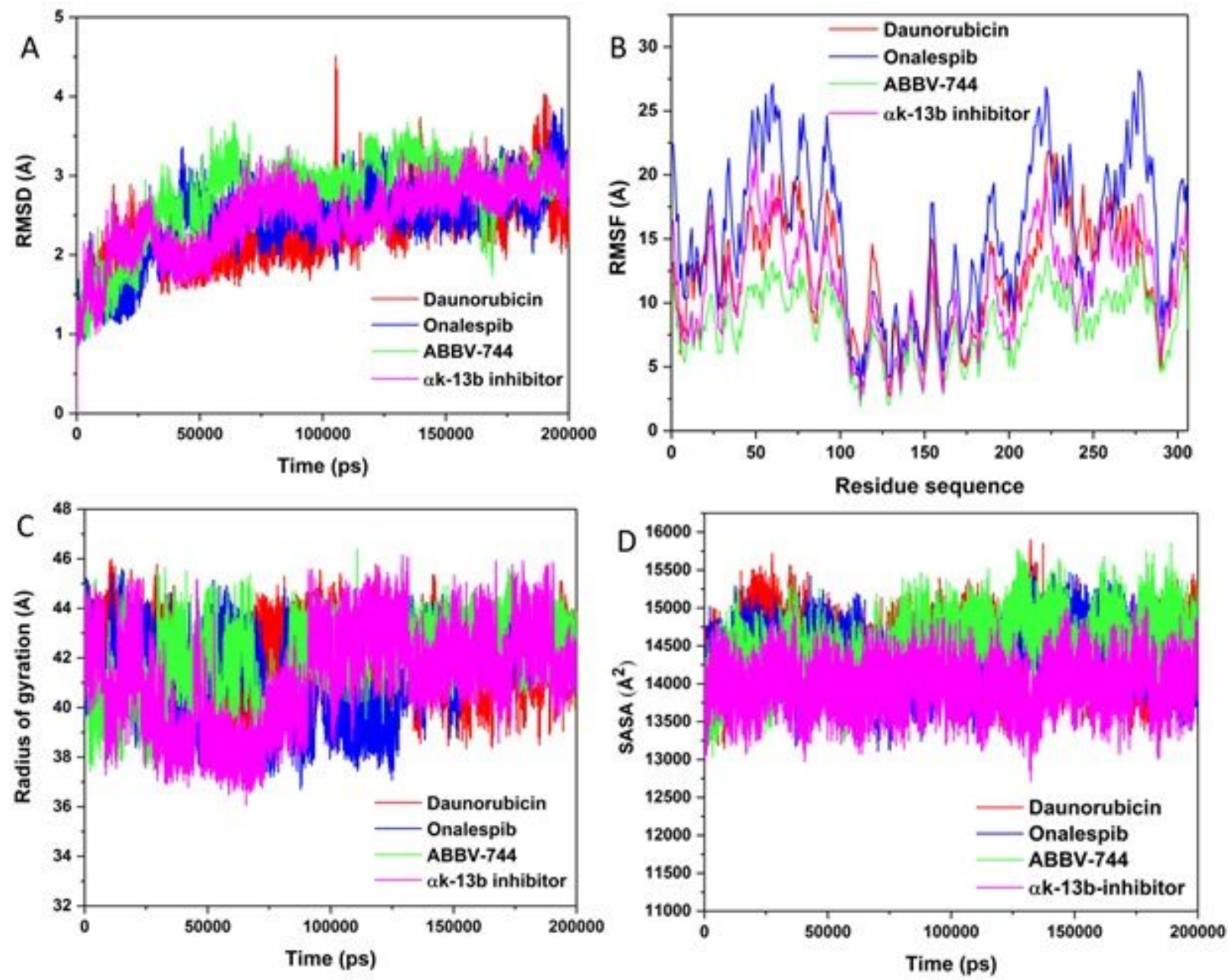

\section{Figure 5}

Structural dynamics of Mpro enzyme-ligand complexes (Daunorubicin in red, Onalespid in blue, ABBV744 in green and $\square k-13 b$-inhibitor in magenta) during $200 \mathrm{~ns}$ of MD simulations. A: Ca backbone RMSD in $\AA$ of all the selected compounds bound to Mpro enzyme; B: Values of RMSF in $\AA$ plotted against residue number for all the selected compounds bound to Mpro enzyme $\mathrm{C}$ : Rg values after compound binding, and C: SASA values of Ca backbone atoms.
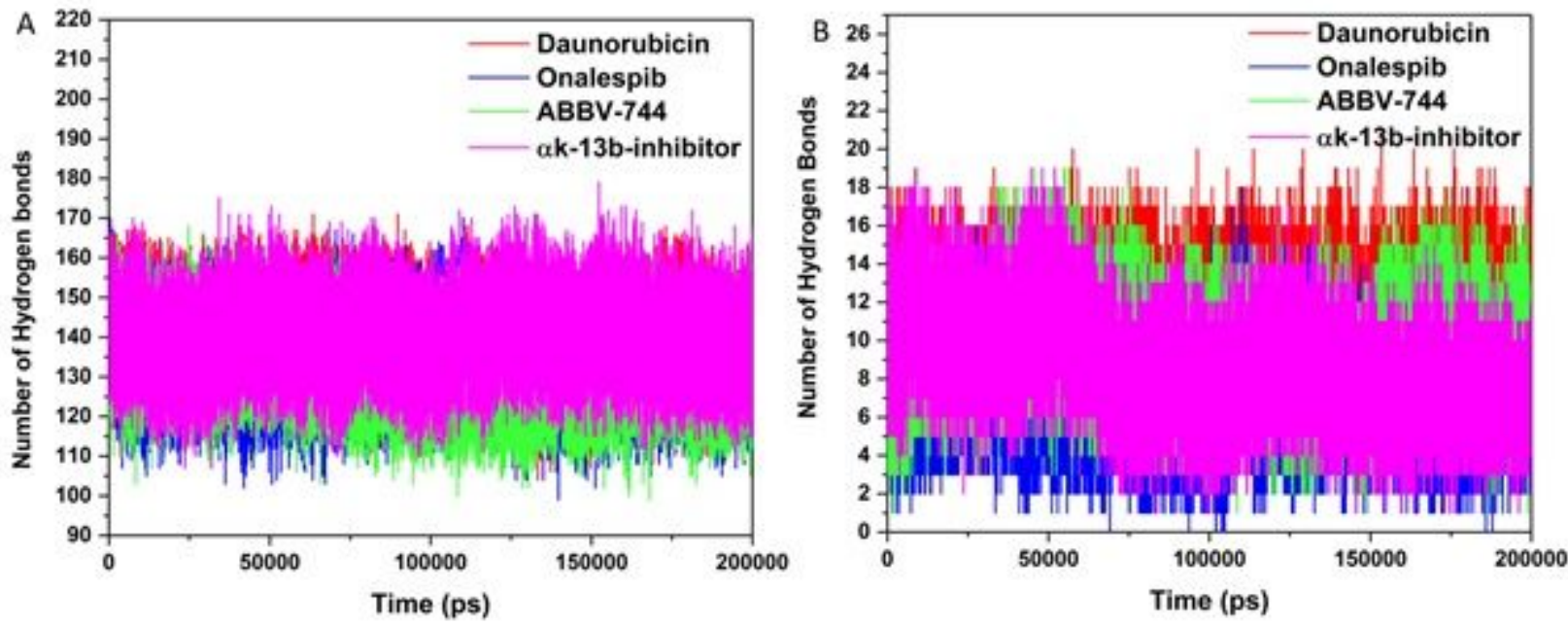
Figure 6

Hydrogen bond analysis. A. Intramolecular and B. Intermolecular hydrogen bonds in Mpro enzyme with the selected compounds (Daunorubicin in red, Onalespid in blue, ABBV-744 in green and Dk-13b-inhibitor in magenta) calculated after 200 ns MD simulation.
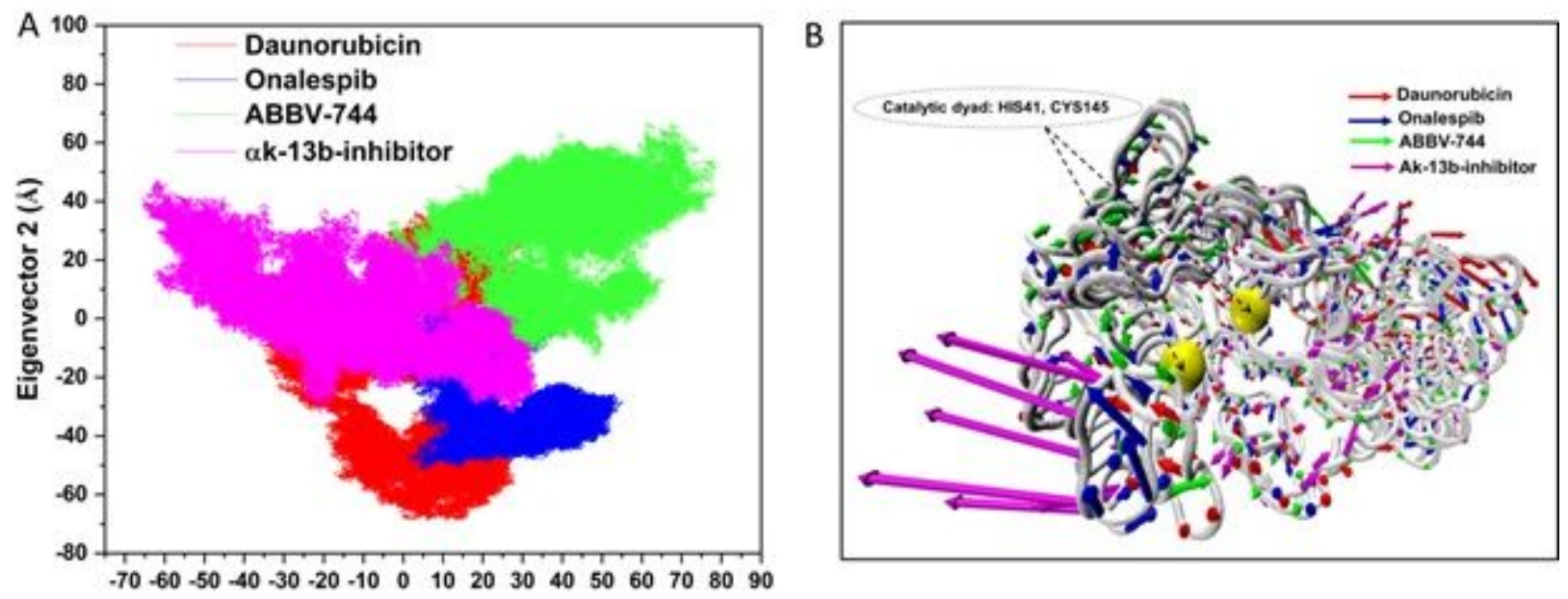

Eigenvector 1 (A)

Figure 7

A. PCA plot constructed by eigenvector 1 vs eigenvector 2 for Daunorubicin (red), Onalespid (blue), ABBV744 (green) and $\mathrm{k}$-13b-inhibitor (magenta) complexes. B. PC1 collective motions for the obtained predominant eigenvectors using principal component analysis over the $200 \mathrm{~ns} \mathrm{MD}$ trajectories for Daunorubicin-Mpro, Onalespid-Mpro, ABBV-744-Mpro and $\square$ k-13b-inhibitor-Mpro.

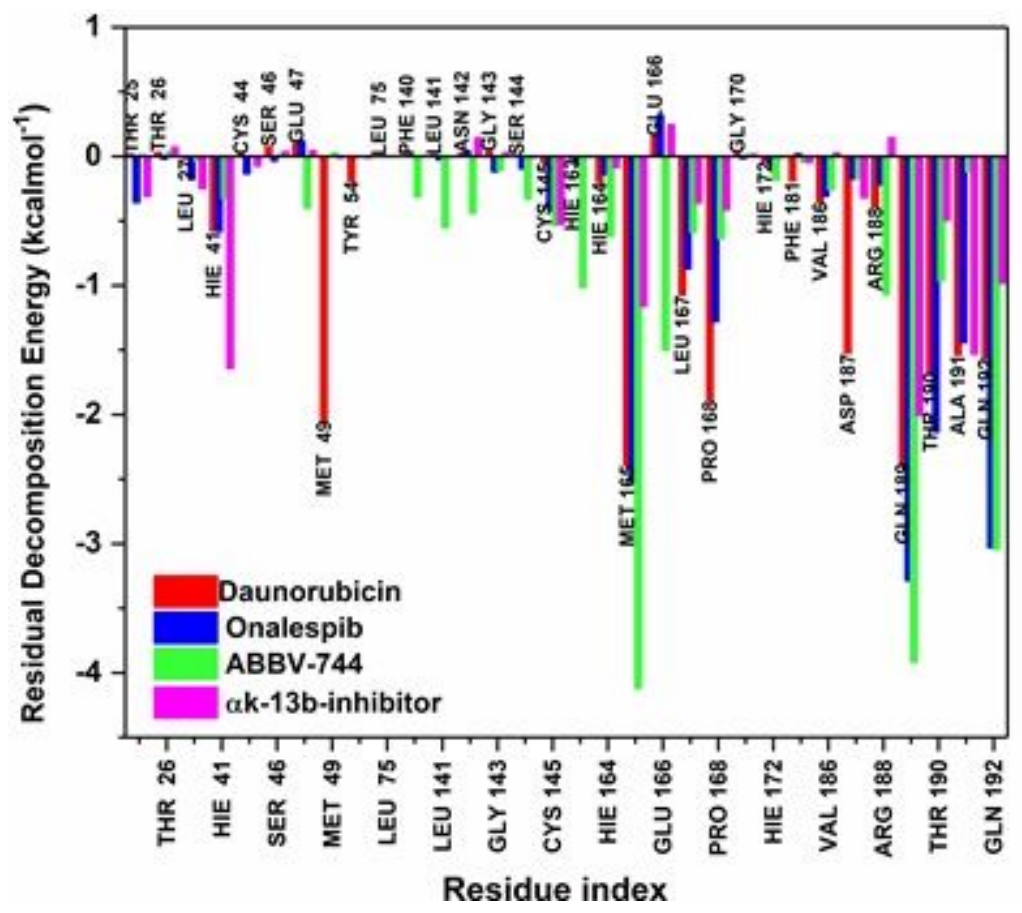

Figure 8 
Per residual decomposition energy of selected compounds (Daunorubicin in red, Onalespid in blue, ABBV744 in green and $\mathrm{Dk}$-3binhibitor in magenta) bound to Mpro enzyme calculated with MMGB/SA approach.

\section{Supplementary Files}

This is a list of supplementary files associated with this preprint. Click to download.

- Equations.pdf

- Scheme1.jpg

- GraphicalAbstract.jpg

- SupplementaryCovid.docx 